\title{
1. Introduction to how to conduct an effective peer review
}

The purpose of this book is to provide a how-to guide for approaching and undertaking peer review of manuscripts submitted to an academic journal in the social sciences, particularly for management domains. Although the expected audiences are early career researchers and doctoral students, we believe that more seasoned academic reviewers may also find some value in this book.

This book materially extends an article we published on reviewing conceptual manuscripts in AMS Review (Griffin and Barczak, 2020). Chapters 2 through 5 of this book, in particular, start from ideas and concepts presented in that article, but expand on them significantly, providing more detail and information than was possible in a shorter journal article. Another difference is that this book focuses on reviewing empirical research, both qualitative and quantitative, rather than concentrating only on conceptual papers. Finally, it also provides advice on later round reviewing (Chapter 11), and information on the future of reviewing (Chapter 12), which also were not covered in the AMS Review article.

Peer review is the process by which experts in a given field of research evaluate and critique the scholarly work of others prior to publication (Allen et al., 2018; Kelly et al., 2014; Pierson, 2015). In the traditional peer review system, "peer" refers to someone who has credentials, typically a Ph.D., in a specific field with a number of years of experience in that discipline (Fitzpatrick and Santo, 2012).

The function of peer review is threefold: to filter out manuscripts of low quality, to improve the quality of manuscripts with potential for publication (Kelly et al., 2014), and to help editors select the work that will be of the greatest interest to the journal's readership (Stiller-Reeve, 2018). Good reviewers identify low-quality manuscripts as those which lack novelty, significant contribution, and/or scientific rigor. Even for manuscripts ultimately rejected from publication consideration, good reviewers provide suggestions about how to improve a manuscript, 
addressing issues such as quality of communication, level of detail, validation of the method, and correction of errors.

Peer review has a long history, dating back to ancient Greece where it is thought to have been used to assess written works generally (Kelly et al., 2014). The first academic journal to formalize peer review was Philosophical Transactions of the Royal Society in 1665 (Elsevier, 2020b; Kelly et al., 2014). However, in a recent article Melinda Baldwin (2018) argues that histories of peer review "have shown that refereeing was not initially thought of as a process that bestowed scientific credibility and that many high-profile journals and grant organizations had unsystematic (or nonexistent) refereeing processes well into the twentieth century" (Baldwin, 2018, p. 539). She further contends that our current perception of peer review as being critical to scientific research only dates back to the Cold War (Baldwin, 2018). Regardless, peer reviewing now has given legitimacy to journals, has become equated with scholarly value, and is used as a means for promoting journals (Tennant, 2018).

The reality today is that the quantity of scientific research across the globe has grown significantly, leading to a greater reliance on peer reviewers by most journals and editors to sort the research of sufficient rigor and quality from that which is not. According to Publons (2018), annual growth in the volume of published articles is 2.6 percent with a corresponding 6.1 percent growth in submissions since 2013. Clearly, with this level of scientific activity, editorial teams are unable (and perhaps unwilling) to make publication decisions on their own. Thus, reviewers play an increasingly important role as gatekeepers helping to determine the quality and credibility of research submitted to scientific journals.

Why is peer review important? In a worldwide survey, 90 percent of respondents indicated that peer review "improves the quality of research articles overall" (Elsevier and Sense About Science, 2019, p. 9). In the same survey, 85 percent agreed that without peer review "there would be no control in scientific communication" (Elsevier and Sense About Science, 2019, p. 5). Similarly, Publons (2018, p. 9) found that 98 percent of respondents view peer review as important or extremely important "for ensuring the general quality and integrity of scholarly communication."

With regard to the level of satisfaction of peer review, the majority of authors are satisfied overall with the process. In their latest survey, the Publishing Research Consortium (PRC) found that 65 percent were satisfied or very satisfied with the peer review system, and that no substantive changes in perceptions of satisfaction had occurred between 
2007 and 2015 (Ware, 2016). In their 2019 survey, Elsevier and Sense About Science found that satisfaction actually increased from 69 percent in 2009 to 75 percent in 2019 (Elsevier and Sense About Science, 2019).

However, criticisms of the peer review system abound. A study published in the Journal of the American Medical Association stated that "Editorial peer review, although widely used, is largely untested and its effects are uncertain" (Jefferson et al., 2002). Some contend that peer review is not effective at detecting errors, plagiarism, or fraud (Kelly et al., 2014). For example, the PRC survey found that only 41 percent and 44 percent of respondents believe that peer review is able to detect fraud or plagiarism, respectively (Ware, 2016). Others argue that the peer review process takes too long (Tennant, 2018), thereby delaying the dissemination of important research findings (Kelly et al., 2014). Some say that peer review prevents researchers from tackling innovative research ideas and questions (Brezis and Birukou, 2020; Kelly et al., 2014) because reviewers tend to be specialized in a certain field and conform to the expectations and values of that field (Brezis and Birukou, 2020). Other criticisms include a lack of clear guidance on how to review (Mulligan et al., 2013; Tennant, 2018) and lack of recognition for performing this largely "volunteer" service (Warne, 2016).

One critique that threatens the sustainability of the peer review process is the claim that only a limited number of competent people are available to review (Kelly et al., 2014). However, using mathematical modeling, Kovanis et al. (2016) show that for 2015 reviewer supply exceeded demand by 15 to 249 percent. But these authors then go on to indicate that the real issue is the imbalance with regard to who is doing the reviewing. It is estimated that 10 percent of reviewers perform 50 percent of all reviews (Publons, 2018). In the biomedical area, Kovanis et al. (2016) estimated that 20 percent of reviewers perform 69 to 94 percent of the reviews. Warne (2016) indicated that 49 percent of reviewers were reviewing for at least five journals.

Another imbalance concern, not surprisingly, is the existence of gender biases in reviewing. Using an algorithmic approach, since most journals and publishers don't collect gender information (Lerback and Hanson, 2017), Publons (2018) has estimated that fewer women than men are involved in peer review. In a study specifically looking at the genders of authors and reviewers from journals of the American Geophysical Union (AGU), Lerback and Hanson (2017) found that women of all ages were underrepresented as reviewers relative to their proportional membership in the AGU. They found that the primary reason for this was that women 
were less likely to be suggested as reviewers by authors and invited to review by editors (Lerback and Hanson, 2017).

There are also regional imbalances in reviewing, with US researchers performing more reviews than the global number of articles published by US authors. In fact, the US dominates in contributing to peer review and, overall, established regions do more peer review than emerging regions relative to their output (Publons, 2018). By contrast, Chinese researchers publish twice as many articles as they review (Warne, 2016). Further, while researchers from emerging areas in general are more likely to accept an invitation to review than are those from developed countries, their reviews tend to be shorter in word length (Publons, 2018).

Finally, and not unexpectedly, early career researchers (ECRs) are also underrepresented in the peer review system as it is not generally viewed as a way to help them develop certain necessary skills (McNair et al., 2019). As we (in Chapter 3) and others contend, however, peer review has significant educational value, particularly to academics early in their research careers. In fact, Jackson et al. (2018) say "peer review is more like a form of pedagogy" (p. 97). Supervisors who receive fewer than four reviews per month were more likely to involve their ECRs in peer review for teaching purposes than those receiving more than four reviews per month (McNair et al., 2019). This same study showed that ECRs agreed that critical thinking and journal style writing were the key learning benefits of peer review (McNair et al., 2019). Another survey showed that almost 54 percent of ECRs had peer reviewed and 65 percent felt they could learn from the mistakes of others (Jamali et al., 2020).

Overall, then, these issues of gender, country of origin, and career-stage reviewer inequality are placing pressure on a small number of volunteer reviewers to deal with the increasing demand for high-quality peer reviews (Kovanis et al., 2016). Seventy-five percent of editors indicate that "finding reviewers and getting them to accept review invitations is the hardest part of their job" (Publons, 2018, p. 27). In fact, the average number of review invitations in 2017 was 2.4 compared to 1.9 in 2013 (Publons, 2018). It is estimated that, at this rate, 3.6 invitations will be needed for each review by 2025 (Publons, 2018).

So, what is the solution? To us, the solution is to help all current (and potential) reviewers by providing guidance on how to do high-quality, constructive reviews as efficiently as possible (Elsevier and Sense About Science, 2019; Spot on Report, 2017; Warne, 2016). In the Publons survey, 88 percent of respondents believe that "peer review training is important or extremely important" while 80 percent believe that "more 
peer review training will have a positive or an extremely positive impact on the overall efficacy of the peer review process" (Publons, 2018, p. 47). The survey by Elsevier and Sense About Science supports these perspectives, with 66 percent indicating that clear guidance needs to be given to reviewers and 37 percent signifying that formal training should be provided (Elsevier and Sense About Science, 2019).

However, our experience as journal editors, and as confirmed by other editors, suggests that few researchers in any discipline are trained in how to do effective peer review (Benos et al., 2003; Rojewski and Domenico, 2005). About 39 percent of respondents stated that they "never received any peer review training" (Publons, 2018, p. 14). In lieu of formal training, researchers undertake the following means to get some type of guidance: self-selected readings, guidelines from journals, Committee on Publication Ethics guidelines, and/or informal advice from supervisors/colleagues (COPE, 2019; Publons, 2018; Warne, 2016). Yet, many researchers are interested in training. According to Warne (2016), 77 percent expressed "an interest in receiving further training" while Publons (2018) found that almost 45 percent of researchers wanted training on how to do a peer review.

In response to the need for training, Elsevier, Publons, Sense About Science, and the American Chemical Society, as well as others, now offer peer review training courses (McDowell et al., 2019), some with certification. ${ }^{1}$ We also offer a one-day in-person Peer Review Workshop for universities and academic associations based on the precepts we have developed for this book and using excerpts and examples from actual journal submissions to generate real-time discussions about how to respond as a peer reviewer. This book follows the general structure and content of our workshop. In both, we use real journal submissions we are familiar with (presented in the Appendices) to provide examples of the types of constructive comments that might be made for each manuscript section.

Chapter 2 provides our definition of a "high-quality review." A short like/dislike/reject review of several lines or one in which the reviewer is promoting their own research is not helpful to editors or editorial teams. They need "high-quality reviews" to make the best and fairest decisions about submitted manuscripts. The chapter breaks down key phrases in our definition and expounds on them to highlight the importance of each. The purpose is to build a foundation for the perspective that all reviewers should take when starting the review process. 
Chapter 3 discusses the benefits of reviewing to reviewers, the "WIIFM." These benefits may be obvious to mature reviewers. However, early career researchers and doctoral students may be surprised by some of them. The downsides of reviewing - that it takes time and cognitive energy - are also acknowledged. Of ongoing interest to all those who think about reviewing - reviewing manuscripts for the journals in your research domains provides great exposure to the newest theories, methods, and developments that may help inform your own research.

The fourth chapter focuses on what we call the 5 Rs of reviewing - the roles, responsibilities, responses, reactions, and respect. We discuss what each " $R$ " means and how each should direct one's reviewing behavior.

The fifth chapter presents a peer review template to help guide the reader through a repeatable process for executing a review and writing a peer review report as well as suggestions for getting started on the review. This chapter then gets a bit personal: it acknowledges that, over time, every reviewer will develop their own process and response "style," and we both convey our process and our perception of our individual styles. The chapter closes with a checklist of reminder questions about the minor and easily fixable issues (grammar, typos) that reviewers typically provide at the end of their reviews.

Chapters 6-10 dig into each of the major sections of manuscripts: the introduction; theory and literature review; method; results; and discussion and implications. Each chapter provides a list of issues and corresponding detailed questions for explicit consideration in that section. In the text, we expound upon those issues, providing guidance regarding what to look for in each major category of concern for each manuscript section. Where appropriate, differences between what to look for in qualitative and quantitative research manuscripts are highlighted. Using examples of both a qualitative and a quantitative paper (provided in Appendices 1 and 2), each chapter ends with potential reviewer comments and an editorial synthesis of those comments. To render these comments, both authors independently evaluated each section of each paper and wrote their own review of that section, acting as an anonymous reviewer. We did this because, for pragmatic and confidentiality reasons, we could not use the actual reviews of these submitted manuscripts. As each author was a co-author on one of these sample papers (Gloria - social strategy; Abbie - portfolio management) and, thus, likely biased toward the manuscript, the double reviews of each paper allow readers to see how different reviewers assess the same section of a manuscript. 
Chapter 6 focuses on the introduction, which includes the positioning of the paper, the theoretical and literature gaps, and the contribution. Chapter 7 deals with the theory and literature review sections, and, in the case of quantitative papers, hypothesis development. Chapter 8 emphasizes methods issues, including the sample, data collection, data analysis, and issues of reliability and validity. Differences in expectations between qualitative and quantitative research methods are noted. Chapter 9 focuses on the results section and the need for reviewers to evaluate both the textual as well as the tabular and visual presentation of the results to assess their completeness, accuracy, consistency, believability, and meaningfulness. Again, special considerations for qualitative vs. quantitative research results are also offered. Chapter 10 deals with the discussion and implications sections and differentiates between these two components. Specifically, the discussion represents an explanation of the results, which requires contrasting and comparing them relative to previous findings and theory. By contrast, implications focus on the importance of the findings for that journal's audience in terms of theory, practice, and/or policy.

Chapter 11 presents advice pertaining to second, third, etc., rounds of a paper and issues to consider within these subsequent rounds. It focuses in particular on three of the 5 Rs - responsibilities, responses, and reactions - as they pertain to these subsequent revision rounds.

The book concludes with an overview of emerging trends in peer reviewing, differentiating between new approaches that address general critiques of peer review quality and scarcity and those that tackle peer review transparency.

Our journey to writing this book began when we served as Editor and Associate Editor of an innovation journal. We noticed how difficult it had become over the last few years to get even two reviewers for each manuscript, let alone three. Sometimes the Associate Editors would send out eight, nine, or even more invitations to get two people who would accept. Then you just hoped that they actually completed their review - and in a timely manner.

In such instances of desperation to even find a reviewer willing to review, it is difficult, if not impossible, to ensure that each reviewer has the expertise needed for that particular manuscript. So, we initially developed a one-and-a-half-hour workshop for a small academic conference in November 2018. We had 11 registered attendees, but four times that number actually showed up, including a number of eminent, senior 
scholars in our field. At that point, we knew we had hit upon a real need in our academic community.

Next, we developed a one-day workshop that we offered to early career researchers at a German university. We also further developed our shorter workshop for other conferences and delivered it at the Academy of Management in 2019. After the success of several workshops, both shorter and longer, we began promoting our one-day workshop to colleagues at universities, primarily in Europe, and began organizing visits to a number of them. We also wrote and subsequently published an article stemming from our workshop on reviewing for conceptual papers in $A M S$ Review (Griffin and Barczak, 2020). It was from seeing the announcement of our workshop at the Academy of Management Conference in Boston in 2019 that Edward Elgar Publishing contacted us to inquire if we were interested in writing a book on how to carry out peer review. That inquiry and subsequent discussion has led us to this point.

We hope you find this book useful. If so, please pass it on to your colleagues and students.

\section{NOTE}

1. McDowell et al. (2019, pp. 20-21) provide a more extensive list of providers of peer review training courses. 